\title{
Data processing as a critical part of GIS based mapping of renewable energy perspectives
}

\author{
Paul E. Kargashin ${ }^{1}$, Anna I. Prasolova ${ }^{1}$, Boguslav A. Novakovsky ${ }^{1}$, Yulia Yu. Rafikova ${ }^{1, *}$, \\ Roman $V$. Gorbunov ${ }^{2}$ and Tatiana Yu. Gorbunova ${ }^{2}$ \\ ${ }^{1}$ Lomonosov Moscow State University, Department of Geography, Leninskie Gory, 1, Moscow, \\ Russia \\ ${ }^{2}$ T.I. Vyazemsky Karadag Scientific Station - Nature Reserve of the Russian Academy of Sciences, \\ Russia
}

\begin{abstract}
Estimation of optimal location for power plants using renewable energy sources requires big data processing and consideration lots of factors. It is suggested to use geographical information systems based approach to simplify workflow. The result of investigation is a series of maps divided into 2 groups: estimation of natural resources for renewable energy, and evaluation of limitations caused with socioeconomic policy. These maps help us to outline the most suitable as well as restricted places for perspective power plants.
\end{abstract}

\section{Introduction}

Many countries all over the world exploit power plants which use unconventional sources to produce electricity and heat. According to the report "Renewable Energy Statistics 2017" published by the International Renewable Energy Agency (IRENA) [1] there are China, several European countries and the USA among the leaders. During the last 5 years Russia also began active development of renewable energy. Nowadays there are several projects of equipment production for solar and wind power plants on the territory of the Russian Federation and a number of big power stations using renewable energy sources. More intensive development of renewable energy in Russia is constrained due to a huge reserves of oil, coal and gas. Besides the most part of the country has extreme natural conditions which seriously affect the cost and complexity of industrial objects construction. It is also considered that Russia, due to its geographical location, has a few places where solar energy could be utilized.

Despite of these facts the nowadays projects in Altai Region and in Yakutiya Republic [2] show that solar energy can be utilized effectively even in northern climatic conditions. These two cases proved, that it becomes crucially to develop scientific approaches which can be used to estimate the suitability of territories for renewable energy utilization. It means that efficiency depends not only on the parameters of the power plant but also on the right choice of its location. These complex research have to deal with a wide row of data and have be spatially oriented.

\footnotetext{
${ }^{*}$ Corresponding author: ju.rafikova@gmail.com
} 
Our investigation is based on computer methods of data collection, storage and processing to outline the most suitable plots for utilization of renewable energy resources. Geographical Information Systems (GIS) and their particular tools are often used for renewable energy modelling $[3,4,5,6]$. It is a common practice to estimate gross potential of different energy sources for the territory and represent the results on the maps. It is obvious that not all the territory suits for energy production. It was suggested that application of GIS and its particular tools would allow to find plots, estimate its suitability and choose the most efficient technology. Each research in science or engineering requires a huge amount of data and it is often a constraint for project results application. Moreover, the available data is usually given in general, but it is important to consider peculiarities for researches. It gives a restriction of level of details. Consequently, the additional task is to replenish insufficient data with results of spatial analysis.

So this paper represents an approach which can be used to analyze a territory of regional level and estimate its perspectives for renewable energy utilization.

\section{Methods, territory and materials}

Approach is based on spatial analysis, so the operational unit is a plot which has its location, square and a number of attributes. Each plot has at least two attributes: 1) renewable energy resources, 2) possible constraints. The first point describes the amount of energy which can be produced or gross value. It is sensible to perform evaluation for solar, wind energy, bioenergy and other resources separately. This attribute shows how much energy could be utilized for electricity production and particular values must be associated with plots. In other words, it is the optimal way to describe a positive effect. Source data for such various energy sources differs. It is common to use databases with reanalysis data to study solar and wind energy. One of the most popular databases is NASA Surface meteorology and Solar Energy database [7], the other one is EUMETSAT's Satellite Application Facility on Climate Monitoring (CM SAF) [8]. The described data is located in points or it is spread over the regular network.

Estimation of gross potential of bioenergy is usually based on statistical data. Another way to get data concerning bioenergy is to use space images. Croplands have a specific structure on images and can be easily detected. There is an example of field on the Figure 1. The field differs greatly from the neighboring area. Processing the data from satellites gives us a real area which is used for crops. Area of croplands can be converted to gross potential value through the volume of wastes grown on it.

Besides, there was defined an attribute, which reflects a negative effect. The attribute shows what constraints exist within our research area. The types of constraint differ for various territories. But, there are some universal types: reserved areas, urban areas, forest lands and some others. It is important to take them into account, because it can be restricted by legislation to build industrial objects within such plots. In case of urban areas, it is difficult to find free space to place a power plant. The suggested division and principle of evaluation help to exclude the plots which cannot be used for building.

Methods of spatial data processing are overlay, weighed overlay, data classification, creation of buffers, interpolation, etc. There were used tools of ArcGIS 10 for our work. The software produced by ESRI [9] has a variety of methods to classify all plots. The most perspective plot is that has the highest rate of gross potential value and doesn't have any constraints. It is evidently that the research must be based on different source data: space images, statistical data, maps, climatic databases. Data from all sources was located and associated with different plots. Furthermore, data from different sources was compared to outline perspective areas via maps and GIS tools. 


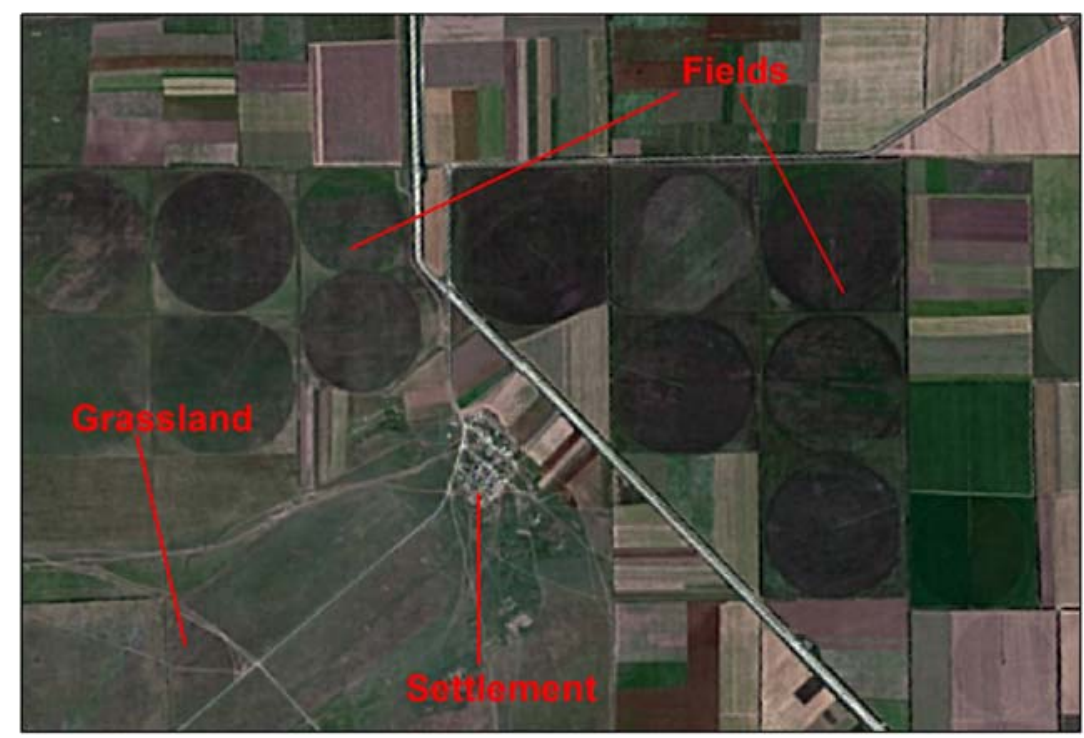

Fig. 1. The example of different types of land use visualization on the space image.

The database of spatial data (BSD) created during the project is implemented in the software product ArcGIS. The geodatabase includes the following datasets:

1) BaseMap - a digital cartographic basis that consists of all elements of a general geographic map (administrative boundaries, hydrography objects, roads, power lines, populated areas, etc.) and supplemented with information on land use and land cover (Land Use/Land Cover). Particular attention in the development of this block was given to Land Use/Land Cover, as it is often these spatial objects that are used to localize thematic information.

2) TempData - a special data set, where the results of intermediate stages of data analysis are stored. This is necessary in order to identify potential errors at the stage of debugging algorithms. This dataset does not have a permanent content and is periodically cleaned.

3) Bioenergy - a set of data that stores information on the volume of agricultural production (imported by a special script via CSV-format), as well as the results of calculating production waste and gross energy potential. In the formation of this set of data, information on different years and different branches of agriculture is separated by separate feature class. If a joint analysis is required, the data is combined by means of queries to the BSD.

4) Gidroenergy - contains the location of gauging stations and the available hydrological characteristics.

5) Solar - a set of data reflecting the necessary information received from weather stations and CM SAF. The above is the essence of the algorithm, which generates point objects from files of the NetCDF format. It is these objects with the necessary attributive data that are imported into the BPD under a unique name containing information about the thematic indicator and date. Similarly, data from weather stations are presented. Thus, each indicator for a certain date is stored in a separate feature class, and SQL queries are used to analyze the time series.

6) Wind - contains information needed to assess wind potentials. The storage principle is the same as for the Solar data set. 
Also in the geodatabase are: - a set of tools (based on Python scripts) that provides data import, the calculation of thematic indicators, analysis of information, etc.; - raster files that cannot be included in the Feature Dataset (software restriction), but are necessary for work. These are space images and a digital relief model. It is not included in the geodatabase, but it is an integral part of the geoinformational support, a folder containing templates for creating thematic data. In the course of the work, the most successful scales, the notation systems were retained for use in the next stage of the project work. Thus, the key approach in the formation of BSD is the maximum fragmentation of information. This is to some extent consistent with the normalization process described in the classic works on relational databases. The approach used allows you to access the desired BSD element by the name of this element, without first analyzing its content. This makes it difficult to visualize the content of the created BSD, but it is extremely convenient for automating calculations and forming cartographic works.

Our investigation was provided for Crimean Peninsula. There are some reasons to select the territory for research below. This region has sufficient natural resources for renewable energy development. Croplands occupy a large area. The region lacks the higher level of energy production than they have at the moment. On the other hand, there are several solar and wind power plants within Crimean Peninsula. Their location can be used to verify our methods. Furthermore, the selected region is characterized by a high recreational and environmental importance. This fact means that we have to take into account not only energy needs and technical capabilities, but also the rational use of natural resources with minimal impact on the environment.

\section{Results}

As a result, it was obtained a series of models and maps. They describe the possibility of alternative energy utilization. As it is said before, for each energy source the potential was estimated separately. For example, perspectives of solar energy utilization were estimated with the help of EUMETSAT's CM SAF data. This database gives us different climatic parameters and allows to analyze a sufficient time range. There was counted an average annual duration of sunshine in hours. One of the maps is shown on the Figure 2.

This map allows us to outline the plots where it is enough amount of sunshine for solar power plant. Further this data was merged according to the land use. This map gives us location of populated areas, reserves, recreational areas. All these areas do not suit for power plant construction. As a result of merging there was gained a summary map which shows the most perspective plots. There is a part of the map on the Figure 3. It describes the south-western part of peninsula.

The most suitable places are marked with yellow. All these plots are located nearby the settlements but outside the populated area. There was also taken in consideration that coastal zone doesn't suit due to its recreational status. The last statement must be commented. There are some restrictions for construction in the water protection zone according to the Water code of the Russian Federation. The security zone is 500 meters. It is also well known that many cultural heritage sites are located on the southern coast of the Crimean Peninsula. The third reason to exclude even offshore sites for power plants locations is the decrease in aesthetic value for recreational zones. The similar research was performed for wind energy and bioenergy resources. 


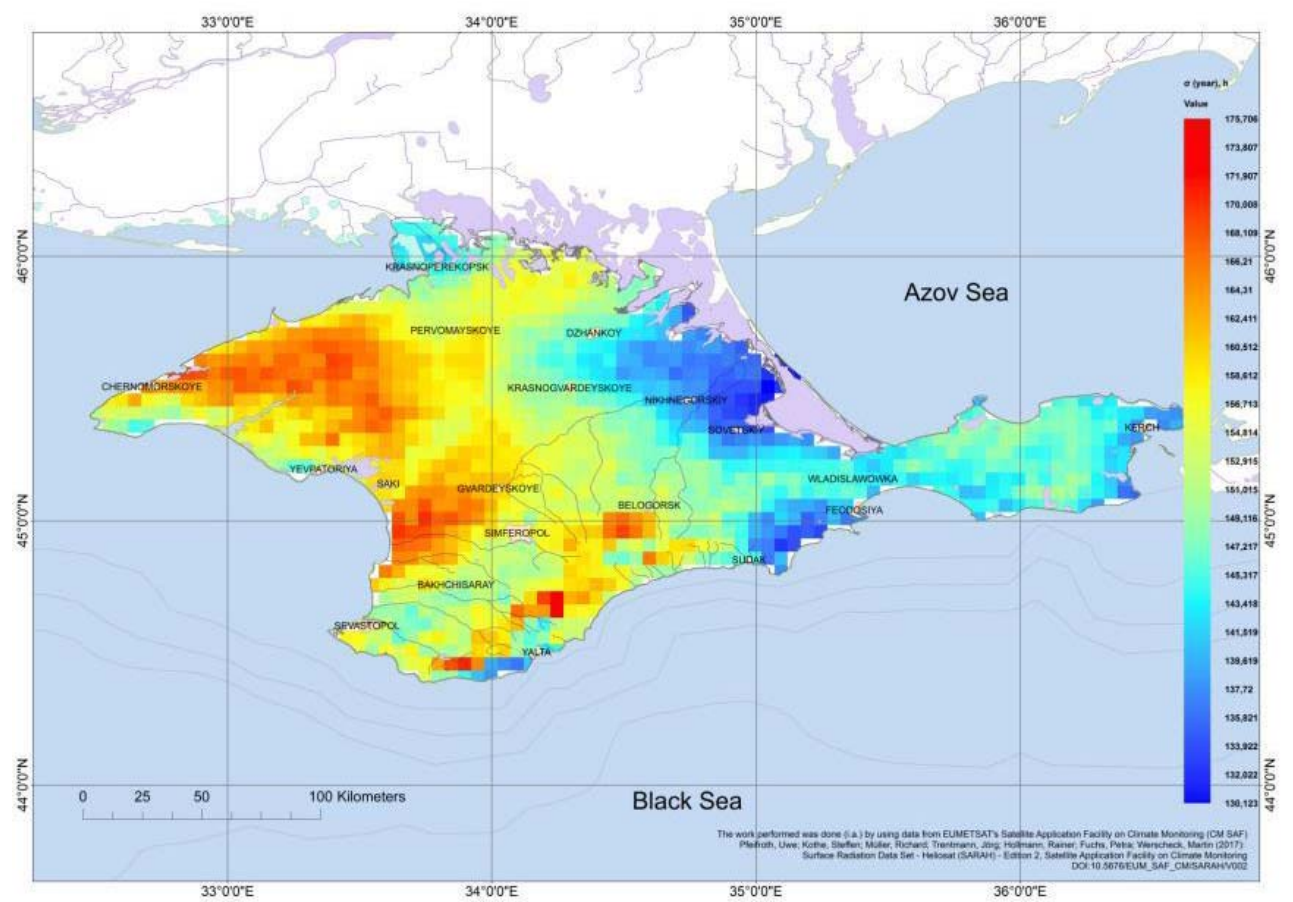

Fig. 2. Map of average annual sunshine duration.

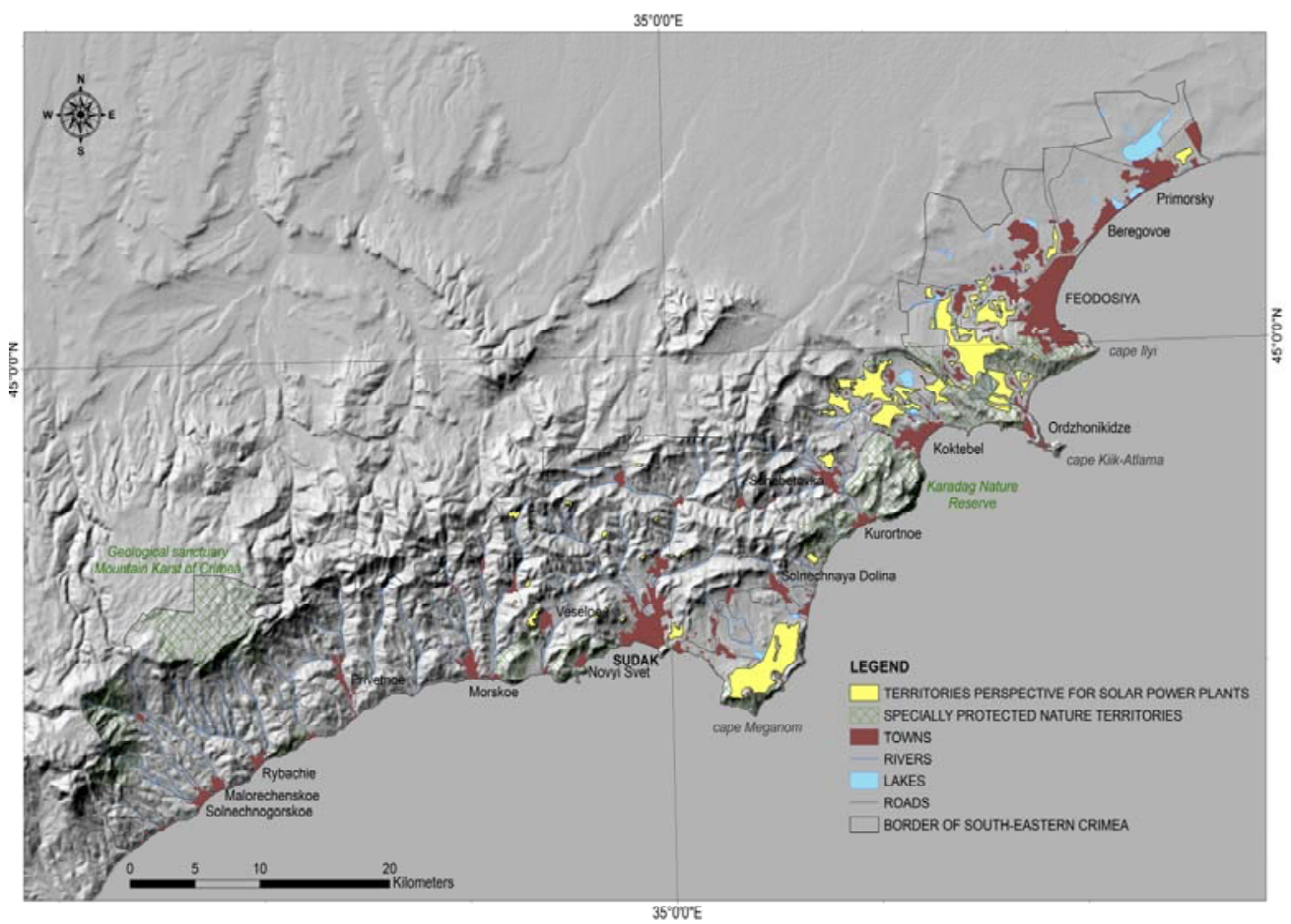

Fig. 3. Suitable places for solar power plants deployment (marked with yellow color). 


\section{Discussion}

As a result of the research there has been gained an understanding how the resources of renewable energy are distributed within the study area. Besides the factors limiting the creation of new objects of renewable energy were identified and systematized. Also there was shown the role of geographic information technologies on each step of investigation

The most valuable part of the work is method of data analysis. The suggested approach was tested on a real data and it was outlined that it can be effectively used for different scales and show a spatial distribution of needful indicators on the maps. Nevertheless, we have to mention some difficulties identified during this work that require further analysis

The method implies an existence of data for each plot of the territory. Only in this case it would be possible to apply the overlay GIS tools to achieve a result of investigation. The most significant problem is to merge data collected from various sources. There were outlined the following issues. At first the only way to compare our data is to associate it with spatial objects, it means that it is necessary to use GIS technologies and geodatabases. Secondly the suggested approach can be applied only for medium scale investigation. Finally, the results show the generalized assessment. It is not suitable for particular research and won't give us value of gross production of solar power plant. On other hand it can be obtained the important information about areas that suit for further investigation and give explanations why some plots don't suit.

Despite of the above-mentioned shortcomings, nowadays the proposed approach is the only available way to take into account the full range of natural, environmental and infrastructure characteristics that significantly affect the possibility of renewable energy projects implementing. The suggested method of investigation could be applied at the regional level and on the pre-project stage within engineering. Possible development of the described ideas is to create and to support the regional electronic atlases of renewable energy resources. Such data representation will help to avoid serious errors in the power facilities planning. In particular, it will be helpfull for those countries and territories where the development of renewable energy is at the initial level and lacks regulatory and information documents.

This work was financially supported by the Russian Foundation for Basic Research (project № 16-0501015 $\backslash 16$ ). The work performed by using data from EUMETSAT's Satellite Application Facility on Climate Monitoring (CM SAF)

\section{References}

1. Renewables 2017 Global Status Report (REN21 Secretariat, Paris, 2017)

2. T.S. Gabderakhmanova, S.V. Kiseleva, S.E. Frid, A.B.Tarasenko, J. of Phys.: Conf. Ser. 774 (2016)

3. B. Sørensen, P. Meibom, Renew. En. 16 (1999)

4. N. Y. Aydin, E. Kentel, S. Duzgun, Renew. Sustain. Energy Rev. 14 (2010)

5. S. Killinger, K. Mainzer, R. McKenna, N. Kreifels, W. Fichtner, Energy, 84 (2015)

6. K. Mainzer, K. Fath, R. Mckenna, J. Stengel, W. Fichtner, and F. Schultmann, Sol. En. 105 (2014)

7. https://power.larc.nasa.gov

8. http://www.cmsaf.eu

9. https://www.esri.com 